\title{
Interoperable Models for Dynamics and Shape Tracking of Soft Fingers
}

\author{
Steeve Mbakop ${ }^{1,2}$, Gilles Tagne ${ }^{1,2}$, Marc-Henri Frouin ${ }^{3}$ and Rochdi Merzouki ${ }^{1}$
}

\begin{abstract}
A lot of works highlight the soft material properties, used in grasping tasks by soft fingers. However, the kinematics as well as the dynamics of these soft fingers are strongly influenced by the material's properties along their soft structure. Due to their compliance, the soft grippers are more often used to achieve form closure grasping which is safer than force closure grasping. One main issue related to soft grasping concerns the shape control of the finger, allowing obtaining perfect compliance when attempting a form closure grasping. In this work, we propose interoperable models based on Pythagorean Hodograph and Euler-Bernoulli's beam dynamics to model both dynamics and shape of Fluidic Elastomeric soft fingers. This makes a relationship between the physical actuators and the virtual control points of the parametric Pythagorean Hodograph (PH) curve which drive the finger shape. The PH curve with its finite control points is used to model and control the kinematics of the shape of the soft fingers, characterized by an infinite degree of freedom (DoF). This modeling will allow controlling the position of the virtual control points of soft fingers. The results of this modeling are validated numerically and experimentally with a soft finger made up of Fluidic Elastomeric Actuators (FEA).
\end{abstract}

\section{INTRODUCTION}

In recent years, advances in materials science applied to mechanics and mechanical design have allowed us to propose better alternatives for robotic manipulations [1]. However, identifying and controlling the optimal shape of soft robots present a challenging topic. This requires some form of actuation computation of the soft robot gathering conventional control methods and motion planning.

The conventional control methods mainly focus on computing control that is based on the desired tip movement. Several methods based on the Jacobian have been largely investigated [2] [3]. Due to the complexity of the task, model free-based techniques [4] have been proposed to inverse the kinematics of the continuum robots. Such local control methods which consider only the tip pose, cannot be suitable to compute motions that require a global control of the overall shape of the soft robots.

On the contrary, motion planning [5] enables the soft continuum manipulators to exhibit a global shape according to a constrained environment. The literature is largely dominated by the probabilistic approaches, which are sampling-based techniques; namely the Rapidly-Exploring Random Graphs

\footnotetext{
${ }^{1}$ Univ. Lille, CNRS, Centrale Lille, UMR 9189 - CRIStAL - Centre de Recherche en Informatique Signal et Automatique de Lille, F-59000 Lille, France. rochdi.merzouki@univ-lille.fr

${ }^{2}$ Junia, Smart Systems \& Energies (SSE), F-59000 Lille, France. steeve.kamga-mbakop@junia.com, gilles.tagnedjunia.com

${ }^{3}$ Niryo, 165 Avenue de Bretagne, 59000 Lille, France. m. frouin@iryo.com
}

(RRG) [6], the Rapidly-Exploring Random Trees (RRT) [7] and the Probabilistic Random Roadmap (PRM) [8]. However, to implement a global shape control of the soft continuum robots, an accurate model that maps the dynamic inputs (physical input) to the geometry of the structure is more than essential.

The real-time shape computation techniques are still lacking in the literature. Some works such as [9], suggest using learning approaches. The present paper aims to investigate a model-based approach. Furthermore, a curvature dynamics feed-back control has been proposed in [10] to track a reference posture curvature. However, the lack of a relationship between the physical input and the adaptive robot shape makes real-time implementation [5] difficult. In [2], a modelbased technique to control the pose of a soft-continuum arm robot is suggested. But, the authors' investigation is only limited to the tip's small displacement.

In this paper, to map the soft finger shape to its physical input, an FEA dynamic model based on Euler-Bernoulli's beam modeling is proposed. The large deflections hypothesis are considered. This dynamic model is interoperated with $\mathrm{PH}$ curves based on prescribed length. This is to model the shape of a soft finger, from a finite number of its $\mathrm{PH}$ virtual control points. Also, one of the features of the $\mathrm{PH}$ parametric curves is to enable inverting the soft finger dynamics.

The paper is organized as follows: In the second section, the fundamentals of FEAs modeling based on EulerBernoulli's dynamics are largely investigated. The interoperable approach which maps the input pressure to a specific finger posture is presented in the third section. These latter allows capturing the relation between the physical actuation and the finite virtual control points of the curve describing the FEA shape. After simulating and experimenting with the proposed approach for validation in the fourth and the fifth section successively, conclusions and prospective works are finally discussed in the last section.

\section{Euler Bernoulli Modeling OF Fluidic ELASTOMERIC SOFT FINGER}

This paper aims to establish the relationship between the control input of a Fluidic Elastomeric soft finger (input pressure $P_{i n}$ ) and its shape curve. For this purpose, an approach based on parametric $\mathrm{PH}$ curves is used for kinematic modeling of the shape, where the adaptive position of $\mathrm{PH}$ control points is deduced from the Euler Bernoulli dynamic model. 


\section{A. Actuation modeling}

The dynamic bending of a fluidic elastometic soft finger is created from the compressed air pressure trapped in the chamber at the tip (free end) of the actuator [11]. Knowing that the static configuration of the soft finger corresponds to the initial air pressure in the chamber, which makes the equilibrium with the gravity effort.

Let us define: $A=a^{2}$, the area of the cross-section of the actuator, $v$, the size ratio of the actuator, $b$, the width of the cross-sectional area and $H_{e}$, the height. The normal force generated (see Fig. 1) by the input pressure on each horizontal line of the actuator tip, being at a distance of $h$ from the bottom layer is given as follows:

$$
\mathrm{d} F=\left(v a P_{i n}\right) \mathrm{d} h,
$$

where $P_{i n}$ denotes the input pressure. $A$ and $v$ are defined as follows:

$$
v=\sqrt{\frac{b}{H_{e}}}, \quad A=b H_{e} .
$$

$\mathrm{d} F$ is the infinitesimal normal force acting on the crosssectional area due to the air pressure along with the infinitesimal distance $\mathrm{d} h$.

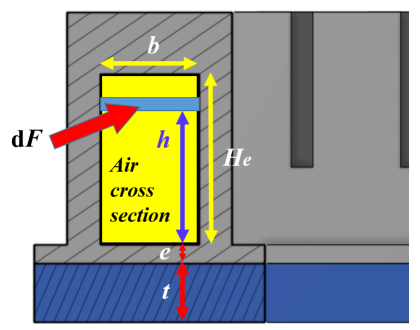

Fig. 1: Effort modeling at the cross-section

Then, the bending moment is deduced as follows:

$$
M_{e}=\int(h+(t+e)) \mathrm{d} F=\int_{0}^{H_{e}}(h+(t+e))\left(v a P_{i n}\right) \mathrm{d} h,
$$

where $t$ and $e$ are respectively the thickness of the inextensible layer and the thickness of the air channel, as presented in Fig. 1. Assuming one control input $P_{\text {in }}$ for a given portion, the bending moment that acts on the soft structure can be calculated by considering its value at the tip. It is perceptible that the bending moment $M_{e}$ is proportional to the input pressure $P_{i n}$ by a geometric constant $\Psi$.

$$
M_{e}=\frac{0.5 a+(t+e) v}{v} a^{2} P_{i n}=\Psi P_{i n} .
$$

\section{B. Euler Bernoulli dynamics for soft finger}

In the following development, it is assumed that the soft finger length remains constant. For modeling purpose, a soft finger is viewed as a thin cantilever planar beam. This modeling is considered for the case of an under-actuated soft finger robot with a high degree of freedom, whose bending is due to the moment caused by the fluid trapped at the free end of the structure. Therefore, the soft finger is supposed to have a moment at the tip [12] [11].

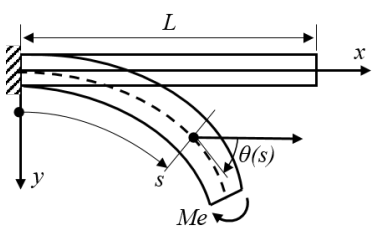

Fig. 2: Planar beam modeling of soft finger

Let us consider a thin cantilever beam of length $L$ subjected to a moment $M_{e}$ which is applied at the free end of the beam, as shown in Fig. 2.

We denote $s(0 \leq s \leq L)$ as the curvilinear coordinate along the axial line, measured from the clamped end, $\theta(s)$ the angle between the positive $x$-axis and the tangent to the neutral axis at point $s$ (Fig. 2). In addition, let $M$ denotes the bending moment at the location $s$, and $\mathrm{d} M$ its infinitesimal variation (See Fig 3).

An infinitesimal element of the deflected beam is shown in Fig. 3.

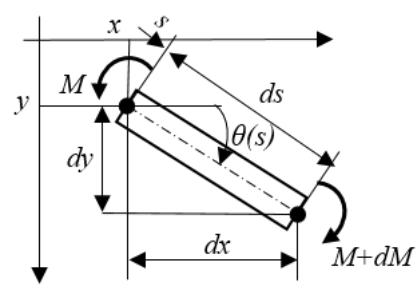

Fig. 3: Infinitesimal element

The equilibrium of bending moments yields:

$$
\mathrm{d} M=0,
$$

where the boundary conditions for the structure are:

$$
\begin{gathered}
\theta(s=0)=0, \\
M(s=L)=M_{e} .
\end{gathered}
$$

Additionally,

$$
\begin{aligned}
& x(s=0)=0, \\
& y(s=0)=0 .
\end{aligned}
$$

According to Euler-Bernoulli (EB) beam dynamics for large deflection, the planar bending moment at each point of the beam is described as follows:

$$
M(s)=\left(E+P_{\text {in }}\right) I \frac{1}{R(s)} .
$$

where $I$ is the static moment of inertia. $\left(E+P_{\text {in }}\right) I$ being the variable flexural rigidity of the inflated beam [13]. The curvature $\kappa(s)$ is described as:

$$
\kappa(s)=\frac{1}{R(s)}
$$

$R(s)$ is the local radius of curvature.

The above development is consistent with the end moment due to a single dynamic input that acts on the soft structure. 
By considering Eq. 4, it is stated from EB beam dynamics that the curvature $\kappa(s)$ of the structure can be expressed as follows:

$$
\kappa(s)=\frac{\Psi}{\left(E+P_{i n}\right) I} P_{i n}
$$

Regarding to boundary conditions, it is deduced that:

$$
\theta(s)=\frac{\Psi P_{i n}}{\left(E+P_{i n}\right) I} s
$$

It can be seen that the finger's inclination angle is varying linearly along the longitudinal axis. A circular $\operatorname{arch} p(s)$ is obtained. Its first derivative, $p^{\prime}(s)$ reads with:

$$
\begin{aligned}
p^{\prime}(s) & =\left(x^{\prime}(s), y^{\prime}(s)\right), \\
x^{\prime}(s) & =\cos \theta(s), \\
y^{\prime}(s) & =\sin \theta(s) .
\end{aligned}
$$

Note that $p^{\prime}(s)$ is tangent to the curve at point $s$.

$$
\begin{aligned}
& x(s)=\left(\frac{\left(E+P_{\text {in }}\right) I}{\Psi P_{\text {in }}}\right) \sin \left(\left[\frac{\Psi P_{\text {in }}}{\left(E+P_{\text {in }}\right) I}\right] s\right), \\
& y(s)=\left(\frac{\left(E+P_{\text {in }}\right) I}{\Psi P_{\text {in }}}\right)\left[1-\cos \left(\left[\frac{\Psi P_{\text {in }}}{\left(E+P_{\text {in }}\right) I}\right] s\right)\right] .
\end{aligned}
$$

In the case of two physical inputs the shape kinematics can be expressed for two phalanges:

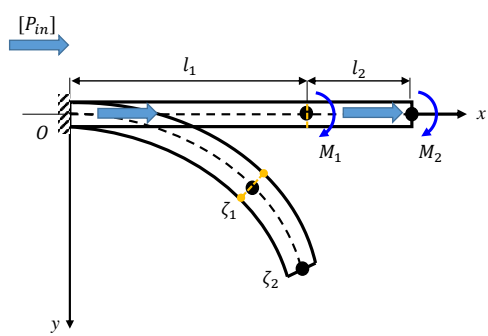

Fig. 4: Geometry features of an under-actuated soft finger

\section{1) First phalange:}

$$
\begin{aligned}
& x_{1}(s)=\frac{\left(E_{1}+P_{1 \text { in }}\right) I_{1}}{M_{1}} \sin \left(\frac{M_{1}}{\left(E_{1}+P_{1 \text { in }}\right) I_{1}} s\right), \\
& y_{1}(s)=\frac{\left(E_{1}+P_{1 \text { in }}\right) I_{1}}{M_{1}}\left[1-\cos \left(\frac{M_{1}}{\left(E_{1}+P_{1 \text { in }}\right) I_{1}} s\right)\right],
\end{aligned}
$$

with

$$
M_{1}=\Psi_{1} P_{1 \text { in }}+\Psi_{2} P_{2 i n}
$$

\section{2) Second phalange:}

$$
\begin{aligned}
x_{2}(s)= & \frac{\left(E_{2}+P_{2 \text { in }}\right) I_{2}}{\Psi P_{2 i n}}\left[\sin \left(\frac{\Psi P_{2 i n}}{\left(E_{2}+P_{2 \text { in }}\right) I_{2}} s+\theta_{1}\left(l_{1}\right)\right)\right. \\
- & \left.\sin \theta_{1}\left(l_{1}\right)\right]+x_{1}\left(l_{1}\right), \\
y_{2}(s)= & \frac{\left(E_{2}+P_{2 \text { in }}\right) I_{2}}{\Psi P_{2 i n}}\left[\cos \left(\frac{\Psi P_{2 \text { in }}}{\left(E_{2}+P_{2 \text { in }}\right) I_{2}} s+\theta_{1}\left(l_{1}\right)\right)\right. \\
& \left.-\cos \theta_{1}\left(l_{1}\right)\right]+y_{1}\left(l_{1}\right),
\end{aligned}
$$

where $\left(E_{1}, I_{1}, \Psi_{1}\right)$ and $\left(E_{2}, I_{2}, \Psi_{2}\right)$ are the Young modulus, the static moment of inertia and the geometrical constant $(\mathrm{Eq}$. 4) respectively related to the first and the second phalange of the soft finger. $P_{1 \text { in }}$ and $P_{2 \text { in }}$ represent the physical inputs acting on the first and the second phalange respectively.

\section{INTEROPERABLE PH-EB MOdELS OF SOFT FINGER}

The interoperable Model of the FEAs shows the relationship between the physical control-input (actuation) and the finite virtual control points of the $\mathrm{PH}$ curve, sufficient to completely describe the shape kinematic changes during the actuation. PH control points are fully defined by the end (clamped and free end) point pose.

For that, let $p_{s}$ and $p_{f}$ describe respectively the clamped and the free end positions of the soft finger with $d_{s}, d_{f}$ their respective directions under actuation (input pressure). From Eq. 11-13, the pose of the free end point is computed while the boundaries conditions are consistent with Eq. 6 and Eq. 7. Henceforth, the following holds:

$$
\begin{array}{ll}
p_{s}=(0,0) & d_{s}=(1,0) \\
p_{f}=p_{f}\left(P_{i n}\right), & d_{f}=d_{f}\left(P_{i n}\right) .
\end{array}
$$

The control points $p_{k}$ of the $\mathrm{PH}$ curve (driving the soft finger posture) are determined with respect to [14].

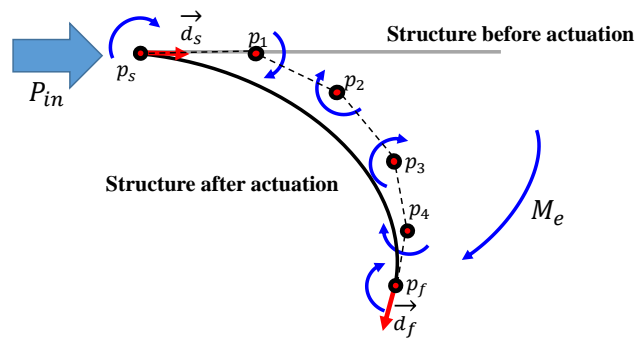

Fig. 5: PH virtual control points

As stated previously (Eq. 15), the EB beam dynamics allows to define the end points pose as a function of the physical input $P_{\text {in }}$.

With the knowledge of the above boundary conditions and the length $L$, the following steps described the $\mathrm{PH}$ quintic formulation, necessary for the shape computation.

\section{A. End tangents of the PH curve of the soft finger}

Let us define $\zeta(0 \leq \zeta \leq 1)$, the normalized curvilinear coordinate along the neutral axis of the soft finger. cooAccording to [14], it exists a complex polynomial function $w$, writing with Bernstein coeficient $w_{k}$ that ensures the existence and the computation of a planar quintics $\mathrm{PH}$ (see Eq. 16). In the considered development, the Pythagorean condition is :

$$
x^{\prime 2}(\zeta)+y^{\prime 2}(\zeta)=\Gamma^{2}(\zeta)
$$

where $\Gamma$ represents the parametric velocity polynomial.

If we considered the following equation:

$$
w(\zeta)=u(\zeta)+i v(\zeta)=\sum_{k=0}^{m} w_{k}\left(\begin{array}{l}
2 \\
k
\end{array}\right)(1-\zeta)^{2-k} \zeta^{k}
$$


where $w_{k}$ are obtained after the integration of the expression:

$$
p^{\prime}(\zeta)=w^{2}(\zeta)
$$

with

$$
w_{k}=u_{k}+i v_{k} .
$$

Thus, the geometric invariant features namely the velocity polynomial $\Gamma$, the unit tangent $d$ and the curvature $\kappa$ at each point of the soft finger are expressed as follows [14] respectively:

$$
\begin{aligned}
\Gamma(\zeta) & =\left|w^{2}(\zeta)\right|, \\
d(\zeta) & =\frac{w^{2}(\zeta)}{\Gamma(\zeta)} \\
\kappa(\zeta) & =2 \frac{\operatorname{Im}\left(\overline{w(\zeta)} w^{\prime}(\zeta)\right)}{\Gamma^{2}(\zeta)}
\end{aligned}
$$

From expression Eq. 20, the tangents $d_{s}$ and $d_{f}$ at end points (clamped and free end respectively) can be derived as follows :

$$
\begin{aligned}
d_{s} & =\frac{w^{2}(0)}{\left|w^{2}(0)\right|}=\exp \left(i \theta_{0}\right), \\
d_{f}\left(P_{i n}\right) & =\frac{w^{2}(1)}{\left|w^{2}(1)\right|}=\exp \left(i \theta_{1}\right) .
\end{aligned}
$$

Hence, by considering:

$$
w_{0}=\operatorname{vexp}\left(i \frac{\theta_{0}}{2}\right), \quad w_{2}=\operatorname{vexp}\left(i \frac{\theta_{1}}{2}\right),
$$

the tangents constraints of Eq. 21 are satisfied. Now, with all the above the following keeps valid:

$$
w_{1}=u_{1}+i v_{1} .
$$

\section{B. PH curve the interpolation under length constraint}

With the knowledge of the end points poses according to the physical input $\left[P_{i n}\right]$, the equations that govern the dynamics of the parametric $\mathrm{PH}$ curve should be carried out.

The parametric velocity polynomial is then formulated with regard to Bézier representation:

$$
\Gamma\left(\zeta, P_{i n}\right)=\sum_{k=0}^{4} \Gamma_{k}\left(\begin{array}{l}
4 \\
k
\end{array}\right)(1-\zeta)^{4-k} \zeta^{k},
$$

$\Gamma_{k}=\Gamma_{k}\left(P_{i n}\right)$ are representing the related Bernstein coefficients, also termed in $P_{i n}$ :

$$
\begin{aligned}
& \Gamma_{0}=\left|w_{0}^{2}\right|, \quad \Gamma_{1}=\operatorname{Re}\left(\overline{w_{0}} w_{1}\right) \\
& \Gamma_{2}=\frac{\left|w_{1}^{2}\right|+\operatorname{Re}\left(\overline{w_{0}} w_{2}\right)}{3} \\
& \Gamma_{3}=\operatorname{Re}\left(\overline{w_{1}} w_{2}\right), \quad \Gamma_{4}=\left|w_{2}^{2}\right|
\end{aligned}
$$

The total arc length (which is a constant $L$ ) of the inextensible layer (neutral axis) of the soft finger yields to:

$$
L=\frac{1}{5} \sum_{k=0}^{4} \Gamma_{k}
$$

By using canonical form of $\mathrm{PH}$ quintic, dynamics of soft finger ends (clamped and free) is ensured trough the following:

$$
\int_{0}^{1} p^{\prime}(\zeta) \mathrm{d} \zeta=1
$$

By using canonical form of PH quintic [14], one can easy demonstrate the following:

$$
2 w_{1}^{2}+3\left(w_{0}+w_{2}\right) w_{1}+3 w_{0}^{2}+3 w_{2}^{2}+w_{0} w_{2}=15 .
$$

Taking in to consideration the inextensible properties of the soft finger, Eq. 26 and Eq. 25 yields :

$$
2\left|w_{1}\right|^{2}+3\left(\overline{w_{0}}+\overline{w_{2}}\right) w_{1}+3\left|w_{0}\right|^{2}+3\left|w_{2}\right|^{2}+\overline{w_{0}} w_{2}=15 L .
$$

Now, when assuming:

$$
\begin{aligned}
\left(c_{0}, s_{0}\right) & =\left(\cos \frac{1}{2} \theta_{0}, \sin \frac{1}{2} \theta_{0}\right), & \left(c_{1}, s_{1}\right) & =\left(\cos \frac{1}{2} \theta_{1}, \sin \frac{1}{2} \theta_{1}\right), \\
\theta_{m} & =\frac{1}{2}\left(\theta_{0}+\theta_{1}\right), & \delta \theta & =\frac{1}{2}\left(\theta_{0}-\theta_{1}\right),
\end{aligned}
$$

and combining Eq. 21, Eq. 28-30, it is possible to compute the Bernstein coefficients $w_{k}$ (Eq. 22) that drive the behavior of the control points $p_{k}$ (see Eq. 34).

Finally, we obtain the following equation system:

$$
\begin{gathered}
4 u_{1}^{2}+6\left(c_{0}+c_{1}\right) u_{1} v \\
+\left(6 c_{0}^{2}+6 c_{1}^{2}+2 c_{0} c_{1}\right) v^{2}-15(L+1)=0, \\
4 u_{1} v_{1}+3\left(c_{0}+c_{1}\right) u_{1} v \\
+3\left(s_{0}+s_{1}\right) v_{1} v\left(6 c_{0} s_{0}+6 c_{1} s_{1}+c_{0} s_{1}+c_{1} s_{0}\right) v^{2}=0, \\
4 v_{1}^{2}+6\left(c_{0}+c_{1}\right) v_{1} v \\
+\left(6 c_{0}^{2}+6 c_{1}^{2}+2 c_{0} c_{1}\right) v^{2}-15(L-1)=0,
\end{gathered}
$$

which describes the dynamics of Bernstein coefficient $w_{k}$ (Eq. 22) that drive the behavior of control points $p_{k}$ (see Eq. 34).

\section{The optimization of the Potential Bending Energy of the curve}

The potential bending energy of the soft finger refers to:

$$
E_{p}=\frac{1}{2} E I \int_{0}^{L}|\kappa(s)|^{2} \mathrm{~d} s
$$

It can be written in its canonical form as follows:

$$
\begin{aligned}
& E_{p}=\frac{1}{2} E I \int_{0}^{1}|\kappa(\zeta)|^{2}|\Gamma(\zeta)| \mathrm{d} \zeta \\
& |\Gamma(\zeta)|=\frac{\mathrm{d} s}{\mathrm{~d} \zeta}
\end{aligned}
$$

The value of $E_{p}$ (see Eq. 33) is optimized according to [14] where the minimum value of $v$ ensures that, $w_{1}$ is minimal trough $u_{1}$ and $v_{1}$. This latter results in minimizing the bending energy of the system with regard to the behavior of a real physical system. 


\section{Computation of the position of the control points}

The minimization of the potential bending energy of the FEAs trough $\mathrm{PH}$ formulation leads to five virtual control points to fully describe the related shape dynamics subject to a physical input $\left[P_{i n}\right]$ :

$$
\begin{aligned}
& p_{1}=p_{s}+\frac{1}{5} w_{0}^{2}\left(P_{i n}\right), \quad p_{2}=p_{1}+\frac{1}{5} w_{0}\left(P_{i n}\right) w_{1}\left(P_{i n}\right) \\
& p_{3}=p_{2}+\frac{1}{5} \frac{2 w_{1}^{2}\left(P_{i n}\right)+w_{0}\left(P_{i n}\right) w_{2}\left(P_{i n}\right)}{3} \\
& p_{4}=p_{3}+\frac{1}{5} w_{1}\left(P_{i n}\right) w_{2}\left(P_{i n}\right), \quad p_{f}=p_{4}+\frac{1}{5} w_{2}^{2}\left(P_{i n}\right)
\end{aligned}
$$

The above control points $p_{k}$ are all termed relative to the input pressure $\left[P_{i n}\right]$ (see Eq. 6, Eq. 7 and Eq. 22).

The shape of the soft structure is recovered with:

$$
p\left(h, P_{\text {in }}\right)=\sum_{k=0}^{5} p_{k}\left(P_{\text {in }}\right)\left(\begin{array}{l}
5 \\
k
\end{array}\right)(1-\zeta)^{5-k} \zeta^{k}
$$

where $p_{k}=p_{k}\left(P_{i n}\right)$

\section{Simulations}

In the following section, the proposed PH-EB modeling is validated numerically compared to Ansys modeling. To do that, two scenarios have been considered. One with a single control input of soft finger, the second with two independent control inputs of two phalanges soft finger robot.

\section{A. Soft finger Design and finite element analysis}

A FEA like actuator made up of Agilus30 soft material was designed (see Fig. 13). Tensile testing on Agilus30 samples are performed using Instron. A general polynomial form (Eq. 36) for incompressible material is adopted to characterize the material.

$$
W=\sum_{i=1}^{3} C_{i}\left(I_{1}-3\right)^{i}
$$

$W$ denotes the strain-energy density function, $C_{i}$ and $I_{i}$ represent the material constants. $C_{i}$ were determined by fitting the model to uniaxial tensile test data using Ansys Software fitting process. They are used to calculate Young's modulus based on the Yeoh hyperelastic material model:

$$
2 C_{1}=\mu
$$

and obviously,

$$
E=2 \mu(1+v) \text {. }
$$

The FEA's properties are illustrated in Tab. I.

TABLE I: FEAs properties

\begin{tabular}{l|l}
\hline FEA constant & Values \\
\hline$L$ (black soft finger) & $116 \mathrm{~mm}$ (soft finger Length) \\
$l_{1}$ (white soft finger) & $63 \mathrm{~mm}\left(1^{\text {st }}\right.$ portion Length) \\
$l_{2}$ (white soft finger) & $56 \mathrm{~mm}\left(2^{\text {nd }}\right.$ portion Length) \\
$E$ (black soft finger) & $0.36 \mathrm{Mpa}$ \\
$E$ (white soft finger) & $0.4 \mathrm{Mpa}$ \\
\hline
\end{tabular}

\section{B. Simulation results}

The validation step consists into two main steps: First, an offline analysis (Section IV-B) is carried out where the proposed $\mathrm{PH}-\mathrm{EB}$ outputs are compared to those of Ansys and to EB beam dynamics. Second, an online analysis (Section V) gives the real experiments. In both cases, the results are compared using a single control input for 'black soft finger' and multiple control input for 'white soft finger'.

Fig. 6 and Fig. 7 shows several soft finger postures according to different values of the physical control input (respectively one and two control inputs). These results are obtained from Ansys and PH-EB. The analysis of the Cartesian errors are described through Fig. 8 and Fig. 9. These errors are evaluated according to the normalized curvilinear coordinates along the soft finger length. In Fig. 8, only one control input is used. Four postures have been studied, starting from low input pressure $\left(P_{i n}=30 \mathrm{kPa}\right)$ to high input pressure $\left(P_{\text {in }}=90 \mathrm{kPa}\right)$.

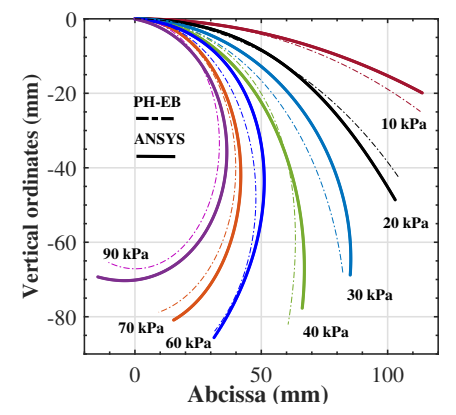

Fig. 6: Simulation of one phalange (black soft finger)

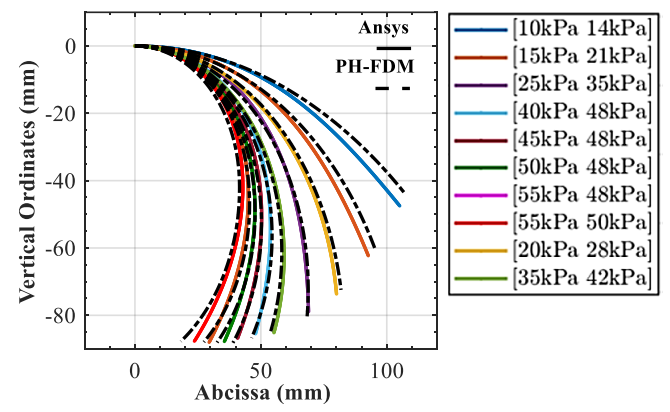

Fig. 7: Simulation of two phalanges (white soft finger)

Within the scope of the simulation, a maximum error of $\Delta_{a_{\max }} \approx 9 \mathrm{~mm}$ can be observed.

Two independent control inputs are involved in Fig. 9. The Cartesian errors has a maximum value of $\Delta_{\text {amax }} \approx 5 \mathrm{~mm}$ (Fig. 9). Fig. 10 and Fig. 12 show that the PH-EB is able to reconstruct accurately the posture got from EB modeling. The figures Fig. 8-Fig. 12 show convergence of the results in-spite of some errors. In addition, it can be observed that the PH-EB tends to lower the error of the prediction (see Fig. 9 and Fig. 11). This corresponds with the optimal bending energy considered in the formulation. However, the accuracy of the model tends to decrease with higher control inputs. 


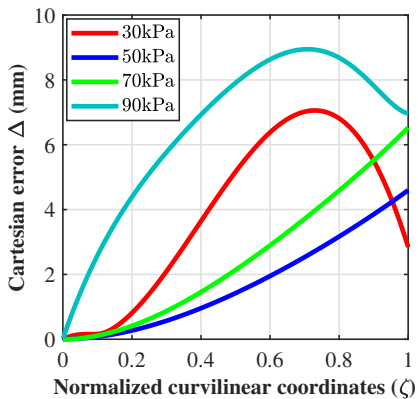

Fig. 8: Errors Ansys/PH-EB of simulated shape reconstruction for one phalange black soft finger

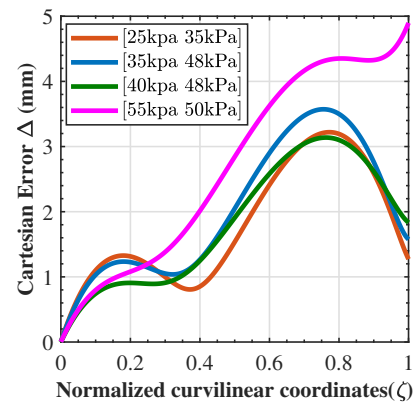

Fig. 9: Errors Ansys/PH-EB of simulated shape reconstruction for two phalanges white soft finger

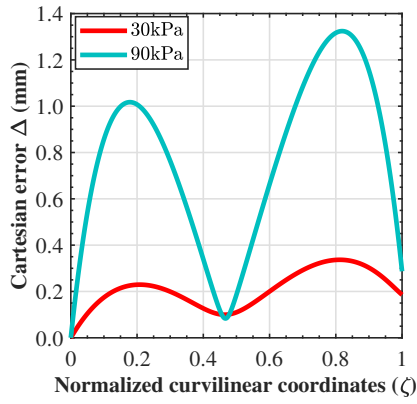

Fig. 10: Errors EB/PH-EB of simulated shape reconstruction for one phalange black soft finger

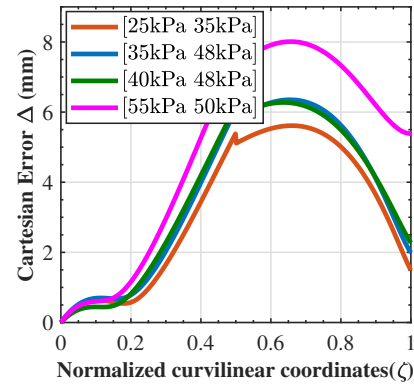

Fig. 11: Errors Ansys/EB of simulated shape reconstruction for two phalanges white soft finger

This is relevant of the fact that, at higher pressures, the EulerBernoulli assumptions may be no longer valid due to non neglectful deformations that occur at finger cross-sections, while there are fully considered by the Ansys computing.

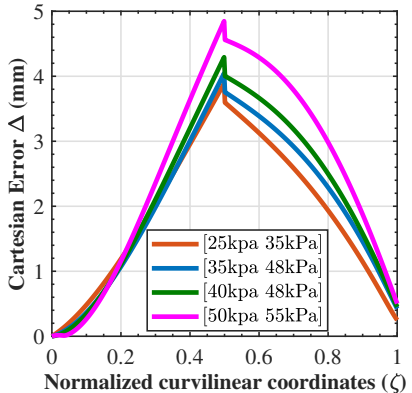

Fig. 12: Errors EB/PH-EB of simulated shape reconstruction for two phalanges white soft finger

\section{EXPERIMENTAL VALIDATION}

The aim of this section is to validate experimentally the soft finger modeling that relies on a finite number of control points.

\section{A. Data acquisition material}

The experiments are carried on 3D printed soft fingers (Fig. 13) using Agilus30 material with different stiffness. Stratagys Connex 500 was used as the 3D printer. The shape and displacements are measured using two different systems: The Optitrack acquisition system and bending sensors are embedded at the bottom of the unextensible layer. The bending sensor of bendlabs brand (1-axis soft flex sensor) is used to capture the angle at the end of finger portions so that the curvature can be assessed. The Simulink Dspace 6 is used to capture real-time data on the curvature given by the sensors while monitoring the convenient air pressure thanks to Festo servo valve. The validation scheme is described on Fig. 14

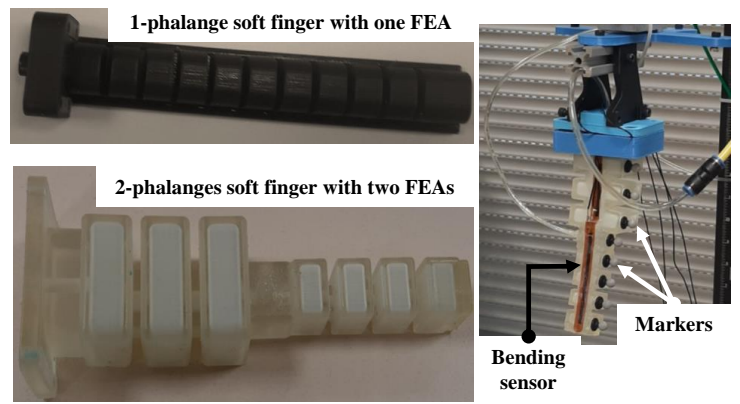

Fig. 13: Black and white soft finger robots

\section{B. Results and discussions}

In the first experiment, the black soft finger robot is actuated from $P_{i n}=30 \mathrm{kPa}$ to $P_{i n}=90 \mathrm{kPa}$. Fig. 15 shows the shape obtained from control points (PH-EB) and the shape tracked with the Optitrack system by using $P_{i n}=30 \mathrm{kPa}$ as single input.

The PH control points are plotted with those of PH-EB. Also, the position of Optitrack markers are plotted (see Fig. 16). 


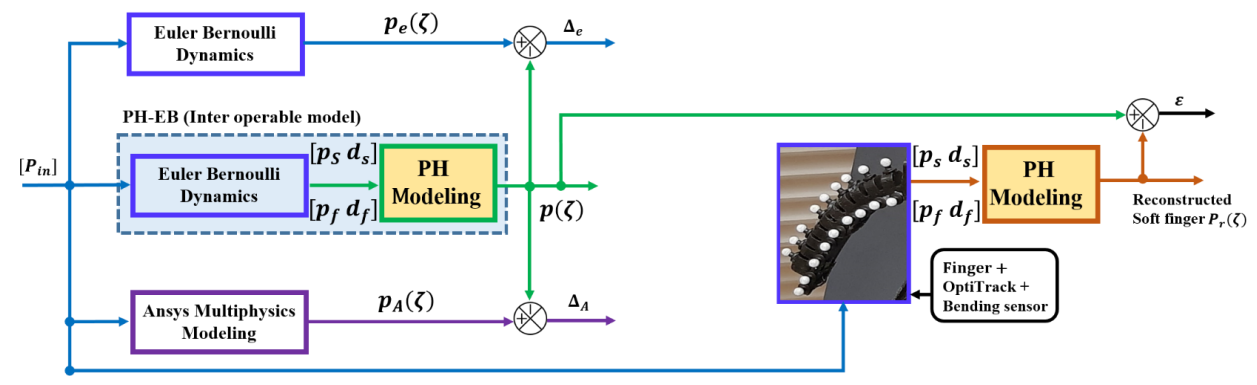

Fig. 14: PH-EB interoperable model validation

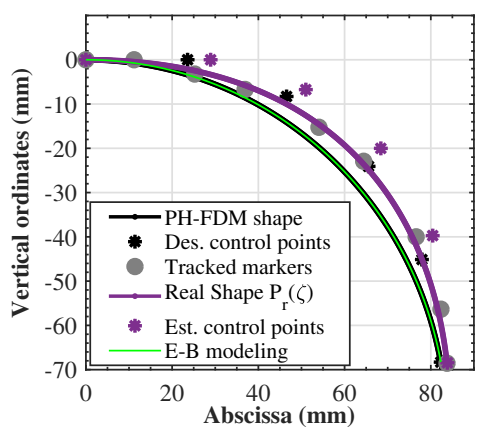

Fig. 15: Shape reconstruction experimental results at $30 \mathrm{Kpa}$

Fig. 15 is showing that the control points are driving the global curvature of the soft finger.

To evaluate the accuracy of the shape compared to the real one, the relative error to the curvilinear coordinates along the soft finger is presented in Fig. 16. It shows a maximum error of $\varepsilon_{\max } \approx 4 \mathrm{~mm}$.

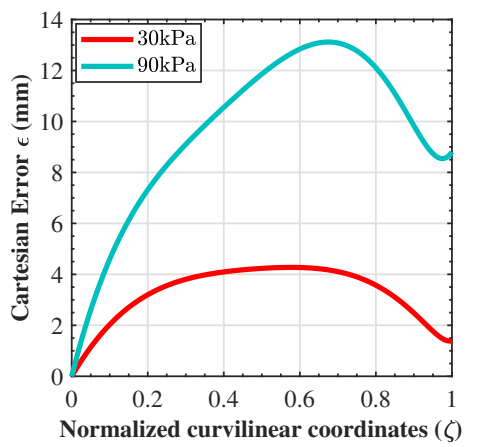

Fig. 16: Errors between PH-EB and real shape for one phalange (black soft finger)

Ansys multiphysical modeling shows $\kappa_{30}^{\prime}=9.1$, while the real tracked curvature is $\kappa=8.9$ and the calculated curvature from PH-EB is about $\kappa_{r 30}=8.5$. Other tests are performed using a single physical input pressure of $P_{i n}=90 \mathrm{kPa}$ to assess the proposed PH-EB modeling. The related Cartesian error is reported in Fig. 16.

In the Fig. 18, a particular case of multiple inputs $P_{i n}=[55 \mathrm{kPa} 50 \mathrm{kPa}]$ is analysed using the white soft finger robot. Only one PH curve has been used to model the phalanges.

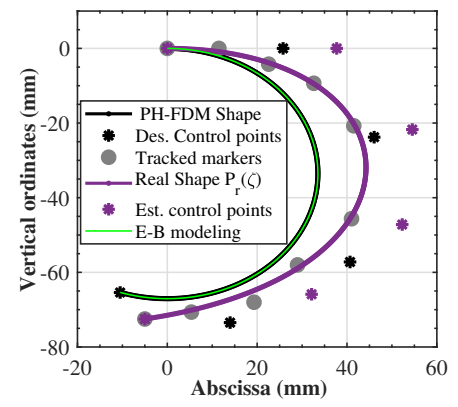

Fig. 17: Shape reconstruction experimental results for $90 \mathrm{kPa}$

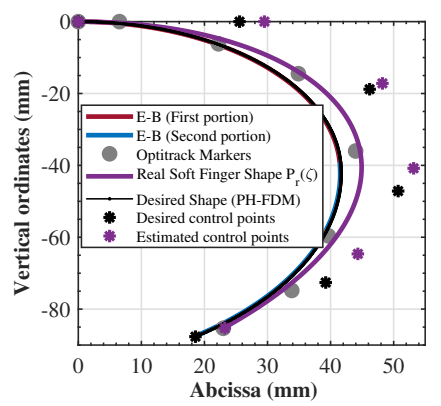

Fig. 18: Shape reconstruction experimental results for $P_{\text {in }}=[55 \mathrm{kPa} 50 \mathrm{kPa}]$

As stated previously, the position of the control points drives the posture of the soft structure to the desired one. To analyse the feature of the PH based modeling, several comparison tests have been made.

The error between the real-time tracked shape and the Ansys multiphysics modeling is presented in Fig. 20 and Fig. 19. It is shown in Fig. 21 that the maximal tracked errors $\varepsilon_{\max } \approx 10 \mathrm{~mm}$. Also, the curvature analysis confirms the $\mathrm{PH}$ curve features for shape reconstruction. Ansys multiphysics modeling shows $\kappa^{\prime}=\left[\begin{array}{ll}26.05 & 18.35\end{array}\right]$ for the first and the second phalanges respectively. It is indicated that the real tracked curvature $\kappa=[25.9478$ 18.2306] compared to PH-EB modeling is $\kappa_{r}=$ [26.3 18.1].

Based on the real experiments, it is shown that Ansys modeling gives better results (see Fig. 20 and Fig. 19) compared to the PH-EB or single EB dynamics (16 and Fig. 21). These results could be explained by the fact that Ansys computing approach takes into account several aspects of the 


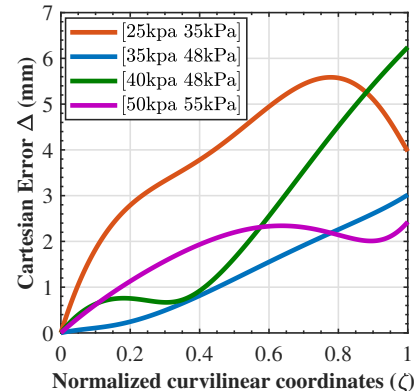

Fig. 19: Errors between Ansys and real shape for two phalanges (white soft finger)

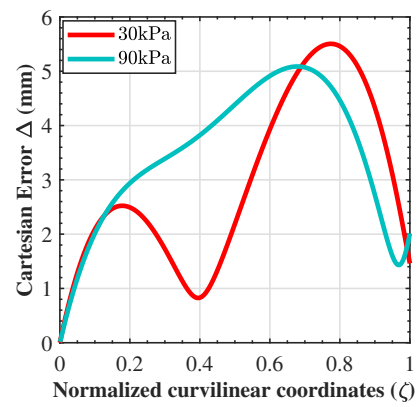

Fig. 20: Errors between Ansys and real shape for one phalange black soft finger

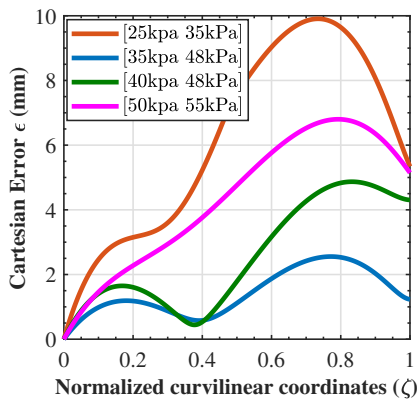

Fig. 21: Errors between PH-EB and real shape for two phalanges white soft finger

finger dynamics such as fluid thermodynamics which is not considered in the frame of the proposed interoperable modeling. Also, the material dilation due to the fluid pressure hasn't been considered. This latter might induces a permanent change of the static moment of inertia which has been taken as a constant in the frame of this work. All these modeling uncertainties lead to some errors compared to real tracked data. However, the proposed PH-EB or EB modeling can be implemented in real-time comparing to Ansys multiphysics modeling. Also, PH-EB indicates an accurate reconstruction of shapes for different postures of soft fingers, generated from on multiple control inputs, comparing to a single EB modeling. This means that with independent controlled phalanges, $\mathrm{PH}$ curve allows an accurate reconstruction for the shape of the overall soft finger robot.

\section{CONCLUSION}

In this paper, an interoperable modeling for Fluidic Elastomeric soft finger robot has been proposed. It is based on properties of Pythagorean Hodograph curve for kinematic shape reconstruction of infinite degree of freedom soft structure and Euler-Bernoulli beam for dynamics modeling of Fluidic Elastomeric Actuators. The aim is to express the relation between the physical inputs of the Fluidic Elastomeric Actuators and the finite control points position of the parametric PH curve of the soft finger. It has been shown that the modeling approach can reconstruct accurately the shapes of the soft finger with different postures, generated from one or multiple physical inputs. It is expected in future works that this model be applied for shape control of soft finger robots for adaptive compliance during the grasping tasks.

\section{REFERENCES}

[1] D. Rus and M. Tolley, "Design, fabrication and control of soft robots," Nature, vol. 521, pp. 467-75, 052015.

[2] A. Ataka, T. Abrar, F. Putzu, H. Godaba, and K. Althoefer, "Modelbased pose control of inflatable eversion robot with variable stiffness," IEEE Robotics and Automation Letters, vol. 5, no. 2, pp. 3398-3405, 2020.

[3] Z. Gong, X. Fang, X. Chen, J. Cheng, Z. Xie, J. Liu, B. Chen, H. Yang, S. Kong, Y. Hao, et al., "A soft manipulator for efficient delicate grasping in shallow water: Modeling, control, and real-world experiments," The International Journal of Robotics Research, 2020.

[4] G. Zheng, Y. Zhou, and M. Ju, "Robust control of a silicone soft robot using neural networks," ISA transactions, vol. 100, pp. 38-45, 2020.

[5] S. Mbakop, G. Tagne, O. Lakhal, R. Merzouki, and S. V. Drakunov, "Path planning and control of mobile soft manipulators with obstacle avoidance," in 2020 3rd IEEE International Conference on Soft Robotics (RoboSoft). IEEE, 2020, pp. 64-69.

[6] L. G. Torres, A. Kuntz, H. B. Gilbert, P. J. Swaney, R. J. Hendrick, R. J. Webster, and R. Alterovitz, "A motion planning approach to automatic obstacle avoidance during concentric tube robot teleoperation," in 2015 IEEE International Conference on Robotics and Automation (ICRA), 2015, pp. 2361-2367

[7] A. Kuntz, A. W. Mahoney, N. E. Peckman, P. L. Anderson, F. Maldonado, R. J. Webster, and R. Alterovitz, "Motion planning for continuum reconfigurable incisionless surgical parallel robots," in 2017 IEEE/RSJ International Conference on Intelligent Robots and Systems (IROS), 2017, pp. 6463-6469.

[8] A. Kuntz, M. Fu, and R. Alterovitz, "Planning high-quality motions for concentric tube robots in point clouds via parallel sampling and optimization," in 2019 IEEE/RSJ International Conference on Intelligent Robots and Systems (IROS), 2019, pp. 2205-2212.

[9] A. Kuntz, A. Sethi, R. J. Webster, and R. Alterovitz, "Learning the complete shape of concentric tube robots," IEEE Transactions on Medical Robotics and Bionics, vol. 2, no. 2, pp. 140-147, 2020.

[10] C. Della Santina, R. K. Katzschmann, A. Bicchi, and D. Rus, "Modelbased dynamic feedback control of a planar soft robot: Trajectory tracking and interaction with the environment," The International Journal of Robotics Research, vol. 39, no. 4, pp. 490-513, 2020.

[11] P. Polygerinos, Z. Wang, J. Overvelde, K. Galloway, R. Wood, K. Bertoldi, and C. Walsh, "Modeling of soft fiber-reinforced bending actuators," Robotics, IEEE Transactions on, vol. 31, pp. 778-789, 06 2015.

[12] Mihael Brojan, Tomaz Videnic, and Franc Kosel, "Non-prismatic nonlinearly elastic cantilever beams subjected to an end moment," Journal of Reinforced Plastics and Composites, vol. 26, no. 11, pp. 1071-1082, 2007.

[13] C. Wielgosz et al., "Bending and buckling of inflatable beams: some new theoretical results," Thin-walled structures, vol. 43, no. 8, pp. 1166-1187, 2005

[14] R. T. Farouki, "Construction of g1 planar hermite interpolants with prescribed arc lengths," Computer Aided Geometric Design, vol. 46, pp. $64-75,2016$. 